\title{
Maximum Likelihood Estimation of Blood Velocity using Doppler Optical Coherence Tomography
}

\author{
Aaron C. Chan ${ }^{a, b}$, Conrad W. Merkle ${ }^{b}$, Edmund Y. Lam ${ }^{a}$, and Vivek J. Srinivasan ${ }^{b}$ \\ ${ }^{a}$ Department of Electrical and Electronic Engineering, The University of Hong Kong, \\ Pokfulam Road, Hong Kong; \\ ${ }^{b}$ Biomedical Engineering Department, UC Davis, 451 Health Sciences Drive, Davis, CA 95616, \\ USA.
}

\begin{abstract}
A recent trend in optical coherence tomography (OCT) hardware has been the move towards higher A-scan rates. However, the estimation of axial blood flow velocities is affected by the presence and type of noise, as well as the estimation method. Higher acquisition rates alone do not enable the accurate quantification of axial blood velocity. Moreover, decorrelation is an unavoidable feature of OCT signals when there is motion relative to the OCT beam. For in-vivo OCT measurements of blood flow, decorrelation noise affects Doppler frequency estimation by broadening the signal spectrum. Here we derive a maximum likelihood estimator (MLE) for Doppler frequency estimation that takes into account spectral broadening due to decorrelation. We compare this estimator with existing techniques. Both theory and experiment show that this estimator is effective, and outperforms the Kasai and additive white Gaussian noise (AWGN) ML estimators. We find that maximum likelihood estimation can be useful for estimating Doppler shifts for slow axial flow and near transverse flow. Due to the inherent linear relationship between decorrelation and Doppler shift of scatterers moving relative to an OCT beam, decorrelation itself may be a measure of flow speed.
\end{abstract}

Keywords: Maximum likelihood estimation, Doppler optical coherence tomography, Decorrelation.

\section{INTRODUCTION}

A recent trend in optical coherence tomography (OCT) hardware development is to increase the A-scan acquisition rate. ${ }^{1,2}$ However, the minimum detectable Doppler shift is not only determined by the sampling rate, but also by the noise statistics and estimation method. ${ }^{3}$ One cannot measure a Doppler shift lower than the square root of the mean squared error (MSE) of an estimator. While the Doppler shift is a reliable way to quantify axial movement, the power spectral density (PSD) of the signal is broadened by decorrelation, especially in in-vivo situations. This effect increases the uncertainty in the Doppler estimate, and is an inherent property of OCT signals of moving scatterers. In addition, decorrelation is more pronounced for higher flow rates. For flow nearly perpendicular to the OCT beam, there may be a high degree of spectral broadening but a very small Doppler shift. Using a rat blood flow phantom, and intralipid flow phantoms, we show that the decorrelation noise maximum likelihood estimator (MLE) ${ }^{3-5}$ can provide better Doppler frequency estimates ${ }^{6-8}$ than the popular Kasai estimator. ${ }^{9}$ Decorrelation is a direct consequence of either flow or OCT scanning, and hence it must be taken into account in Doppler estimation. However, as decorrelation is proportional to the flow rate, or scanning rate, decorrelation itself may be used to deduce the relative speed of the region of interest with respect to the OCT beam. ${ }^{10}$

Further author information: (Send correspondence to Vivek J. Srinivasan)

Aaron C. Chan: E-mail: cwachan@eee.hku.hk, acwchan@ucdavis.edu

Conrad W. Merkle: E-mail: cwmerkle@ucdavis.edu

Edmund Y. Lam: E-mail: elam@eee.hku.hk

Vivek J. Srinivasan: E-mail: vjsriniv@ucdavis.edu

Optical Coherence Tomography and Coherence Domain Optical Methods in Biomedicine XVIII, edited by Joseph A. Izatt, James G. Fujimoto, Valery V. Tuchin, Proc. of SPIE Vol. 8934, 89342J · (C) 2014 SPIE · CCC code: 1605-7422/14/\$18 - doi: 10.1117/12.2036491

Proc. of SPIE Vol. 8934 89342J-1 


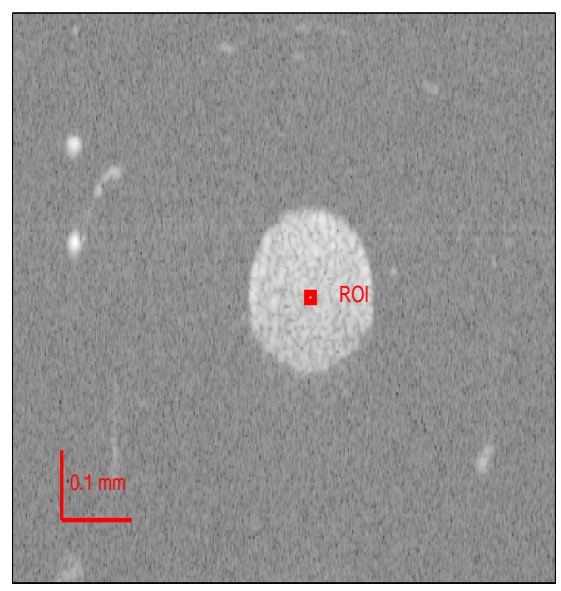

(a) Cross section

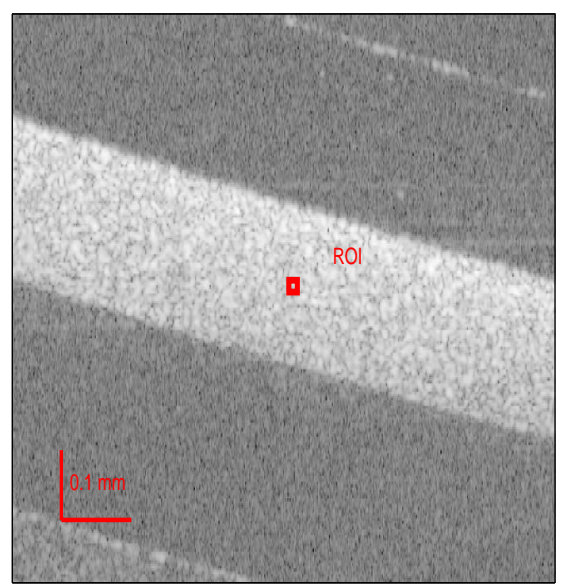

(b) Profile

Figure 1: Cross section and profile of intralipid flow phantom, with no flow. The FEP tubing was tilted at $16.5^{\circ}$ to the horizontal. The region of interest (ROI) was selected to be the center of the tubing to obtain the maximum flow speed.

\section{ADDITIVE WHITE GAUSSIAN NOISE MAXIMUM LIKELIHOOD ESTIMATOR}

If $s_{n}$ is a single data point at time instant $n$, we represent the OCT data for measuring flow velocity as

$$
s_{n}=|r| \exp \left[j\left(n \Omega \Delta t+\phi_{r}\right)\right]+z_{n} .
$$

Here, $|r| \exp \left(j \phi_{r}\right)$ is the unknown complex constant reflectance, and $j=\sqrt{-1}$. The time between measurements is $\Delta t$. The additive noise is given by $z_{n}$, which is circularly symmetric, complex, and Gaussian. The additive white Gaussian noise (AWGN) MLE, $\hat{\Omega}_{\mathrm{MLE}}$, is obtained by choosing the values of the Doppler frequency, $\Omega$, and reflectance phase, $\phi_{r}$, that maximize the real part of the inverse discrete Fourier transform (DFT) of the (complex conjugate of the) signal.

$$
\left(\hat{\Omega}_{\mathrm{MLE}}, \hat{\phi}_{\mathrm{MLE}}\right)^{T}=\underset{\boldsymbol{\Omega}, \phi_{\mathbf{r}}}{\arg \max }\left(\operatorname{Re}\left\{|r| \sum_{n=1}^{N} s_{n}^{*} \exp \left[j\left(n \Omega \Delta t+\phi_{r}\right)\right]\right\}\right) .
$$

As $\phi_{r}$ is chosen to make the summation real, this is equivalent to finding the frequency corresponding to the peak of the PSD. ${ }^{3}$ We showed that the AWGN MLE is best used when the acquisition time is short, and the flow rate is low. ${ }^{3}$ Under these conditions, the noise is well modeled by AWGN, as there is little decorrelation.

\section{DOPPLER SHIFT AND DECORRELATION}

According to theory, ${ }^{11}$ PSD broadening is proportional to scatterer speed for point scatterers. The assumption of point scatterers is valid for intralipid, as the average diameter for intralipid globules is $100 \mathrm{~nm}$, whereas the beam waist of our OCT system was measured to be approximately $7.65 \mu \mathrm{m}$. We define the spatial point spread function of a single scatterer as

$$
h(\rho, z)=\frac{2 K}{\pi w_{\rho}^{2}} \exp \left(-\frac{2 \rho^{2}}{w_{\rho}^{2}}-\frac{2 z^{2}}{w_{z}^{2}}+j \frac{4 \pi n}{\lambda_{0}} z\right),
$$

where we have normalized the function in the transverse plane. The imaginary part of the exponent is due to the Doppler shift. We have chosen to present the point spread function in cylindrical polar coordinates, $(\rho, \phi, z)$, 
as typically OCT beams are circularly symmetric. As the position of moving particles is a function of time, the scatterer pulse response function is obtained by substituting $\rho$ by $v_{\rho} t$ and $z$ by $v_{z} t$ such that ${ }^{11,12}$

$$
p(t)=\frac{2 K}{\pi w_{\rho}^{2}} \exp \left[-\frac{2\left(v_{\rho} t\right)^{2}}{w_{\rho}^{2}}-\frac{2\left(v_{z} t\right)^{2}}{w_{z}^{2}}+j\left(\frac{4 \pi n}{\lambda_{0}}\right) v_{z} t\right] .
$$

The Gaussian shape in the transverse direction, $\rho$, is due to the Gaussian beam profile. The axial point spread function is spectrally shaped in processing to be roughly Gaussian. The OCT signal at one voxel location, $s(t)$, may be modeled as a train of these pulse responses arriving at random times $t_{n}$, with random complex amplitudes $A_{n} .{ }^{13}$ Mathematically, this may be expressed as

$$
\begin{aligned}
s(t) & =p(t) * A(t) \\
A(t) & =\sum_{n} A_{n} \delta\left(t-t_{n}\right),
\end{aligned}
$$

where the power of $A(t)$ is given by $P_{A}$. The temporal autocorrelation function of the OCT speckle pattern may then be expressed $\operatorname{as}^{11,12}$

$$
R(\tau)=\frac{2|K|^{2}}{\pi^{2} w_{\rho}^{4}} \sqrt{\frac{\pi}{\left(\frac{v_{\rho}^{2}}{w_{\rho}^{2}}+\frac{v_{z}^{2}}{w_{z}^{2}}\right)}} P_{A} \exp \left[-\frac{\left(v_{\rho} \tau\right)^{2}}{w_{\rho}^{2}}-\frac{\left(v_{z} \tau\right)^{2}}{w_{z}^{2}}\right] \exp \left[j\left(\frac{4 \pi n}{\lambda_{0}}\right) v_{z} \tau\right],
$$

where the first real exponential represents the decay of the amplitude in time (decorrelation), and the second complex exponential is due to the Doppler shift. The Doppler shift is related to the axial velocity by the formula $v_{z}=\lambda_{0} f_{D} /(2 n)$, where $f_{D}$ is the Doppler frequency. Here $\lambda_{0}$ is the central wavelength of the OCT beam, $v_{\rho}$ and $v_{z}$ are the radial and axial components of velocity of the flow, $n$ is the refractive index, $w_{\rho}$ describes the transverse beam profile (the beam waist), and $w_{z}$ describes the axial resolution (coherence gate). Hence when fitting a Gaussian to the PSD or the absolute value of the autocorrelation function, a plot of the PSD width (reciprocal of the $1 / e^{2}$ coherence time) against the flow speed $v=v_{\rho} / \cos \left(16.5^{\circ}\right)$ would give a straight line plot with a gradient of $\sqrt{\left(\cos ^{2}\left(16.5^{\circ}\right) / w_{\rho}^{2}\right)+\left(\sin ^{2}\left(16.5^{\circ}\right) / w_{z}^{2}\right)}$. The temporal PSD is given by

$$
P(f)=\frac{2|K|^{2}}{\pi w_{\rho}^{4}\left(\frac{v_{\rho}^{2}}{w_{\rho}^{2}}+\frac{v_{z}^{2}}{w_{z}^{2}}\right)} P_{A} \exp \left[-\frac{\pi^{2}\left(f-\frac{2 n v_{z}}{\lambda_{0}}\right)^{2}}{\left(\frac{v_{\rho}^{2}}{w_{\rho}^{2}}+\frac{v_{z}^{2}}{w_{z}^{2}}\right)}\right] .
$$

From this equation one can see that the axial component of velocity gives rise to a Doppler shift, and the transit time of scatterers through the beam determines the amount that the PSD broadens. While it is relatively straightforward to estimate the axial velocity using the Kasai algorithm or an ML method, one would require knowledge of the beam waist, axial resolution and axial velocity to estimate the transverse velocity. Red blood cells (RBCs), however, have diameters of the order of 6 to $8 \mu \mathrm{m}$, which is roughly the same size as an OCT voxel. Hence the point scatterer assumption does not apply, and one would expect that the degree and nature of decorrelation would be different. For RBCs, it is uncertain that the relationship between decorrelation and velocity would obey the theory described above.

We show experimentally using flow phantoms in Fig. 2 that in actual OCT measurements of flow, a Doppler shifted signal is always accompanied by PSD broadening. This broadening may arise due to the scanning of the OCT beam across a region of interest, or the movement of scatterers across a static OCT beam. In Figs. 1-3, a $1325 \mathrm{~nm}$ spectral/Fourier domain OCT microscope with an A-line rate of $91 \mathrm{kHz}$ was used. The spectral bandwidth of this system was inferred to be roughly $83 \mathrm{~nm}$ FWHM from the axial resolution. The axial resolution $w_{z}$ was estimated by fitting a Gaussian to the absolute value of the spatial autocorrelation function of a non-flowing intralipid solution to obtain the $1 / e^{2}$ distance. Prior calibration data on the number of voxels per known vertical distance was used. The $1 / e^{2}$ half-width axial resolution, $w_{z}$ was estimated to be $5.97 \mu \mathrm{m}(7.03$ $\mu \mathrm{m}$ FWHM). Imaging was done on intralipid flow phantoms using $200 \mu \mathrm{m}$ inner diameter fluorinated ethylene propylene (FEP) tubing, which has a refractive index of 1.344. The tubing was submerged in water and tilted 


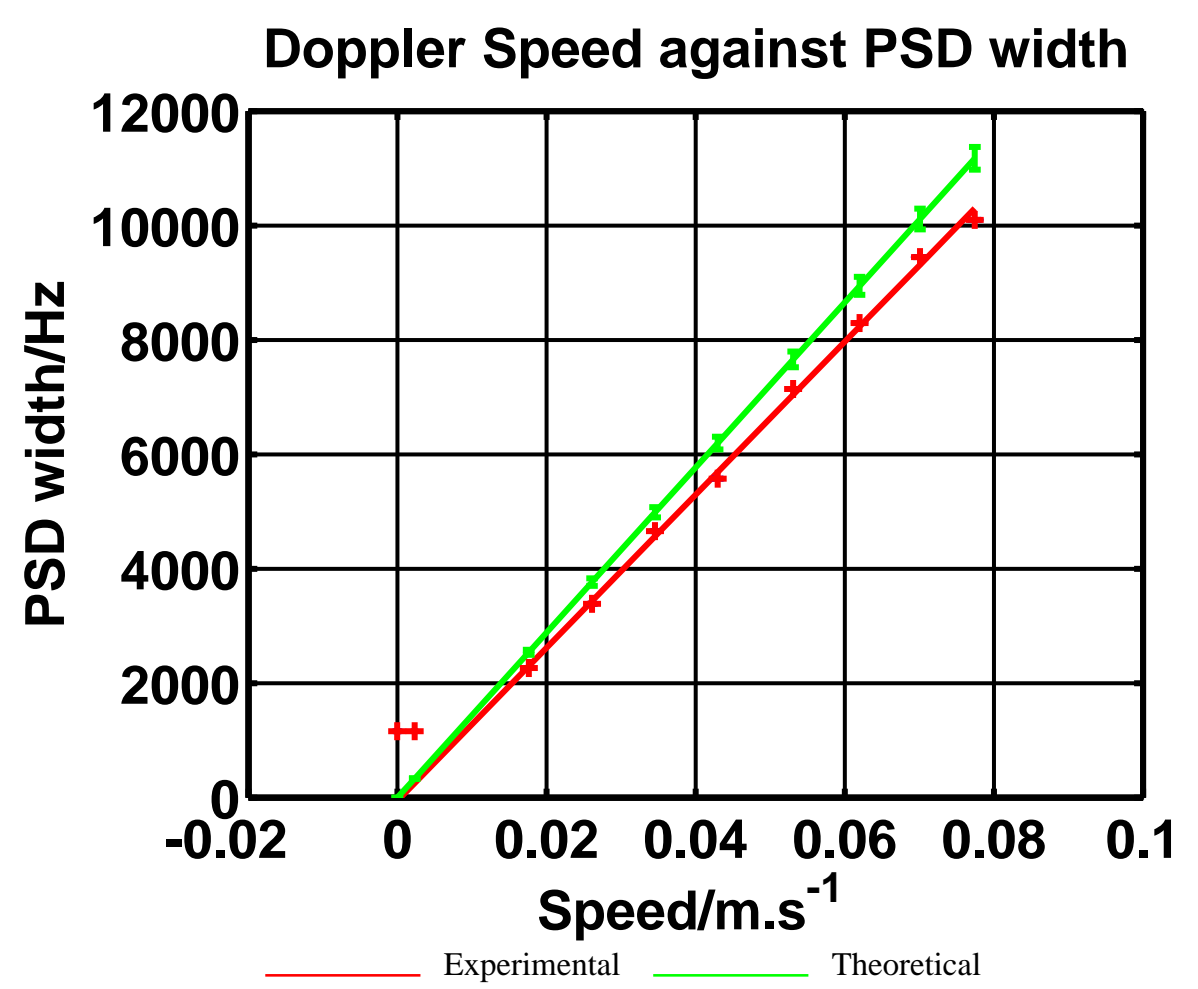

Figure 2: This figure shows that PSD broadening is proportional to flow speed as measured from the Doppler shift. The Dopper shift was estimated with the decorrelation noise MLE, using a data length of 12, and an A-line rate of $91 \mathrm{kHz}$, obtained from intralipid flow through $200 \mu \mathrm{m}$ inner diameter tubing. The tubing was tilted at $16.5^{\circ}$ elevation to obtain a Doppler shift (Fig. 1). M-scans were taken along the center of the tubing, and the region of interest (ROI) was selected to be the center of the tubing to obtain the maximum flow rate. The flow rate was controlled by using a syringe pump. The flow rates were set from 0 to $90 \mu \mathrm{L} \cdot \mathrm{min}^{-1}$.

at $16.5^{\circ}$ elevation relative to the OCT beam to obtain a Doppler shift (Fig. 1). A-lines were acquired from the center of the tubing.

The Doppler speed was measured using the decorrelation noise MLE, using a data length of 12 . The PSD width was calculated by fitting Gaussians to the measured autocorrelations to obtain the $1 / e^{2}$ time, and then taking the reciprocal of this value. Fitting in the temporal domain allows one to exclude data points after a certain time lag, which results in a more accurate fit. The best fit regression line, excluding the first two data points, has a gradient of $1.34 \times 10^{5} \mathrm{~m}^{-1}$, corresponding to an estimated beam waist of approximately $7.65 \mu \mathrm{m}$. The beam waist $\left(1 / e^{2}\right.$ half-width lateral resolution) was measured to be $7.04 \pm 0.14 \mu \mathrm{m}(8.28 \pm 0.16 \mu \mathrm{m} \mathrm{FWHM})$ using a knife edge technique and least-squares fitting of an error function. Using this measured beam waist, theory would predict, from Eq. (6), the gradient to be $1.44 \times 10^{5} \pm 0.03 \times 10^{5} \mathrm{~m}^{-1}$. These results suggest that that in a well calibrated system, the PSD width may be a good measure of flow speed. PSD broadening is an inherent property of any OCT signal with relative motion due to beam scanning or blood flow. Hence for in vivo imaging one would not obtain a Doppler shift without spectral broadening, and the AWGN model is inadequate to estimate the Doppler shift.

Fig. 3 shows the temporal autocorrelation function and PSD of intralipid flowing through FEP tubing at 50 $\mu \mathrm{L} / \mathrm{min}$ as measured from a stationary OCT beam aligned transversely through the center, as shown in Fig. 1. The axial velocity introduces a phase change of the autocorrelation function with time but does not change its absolute value. 


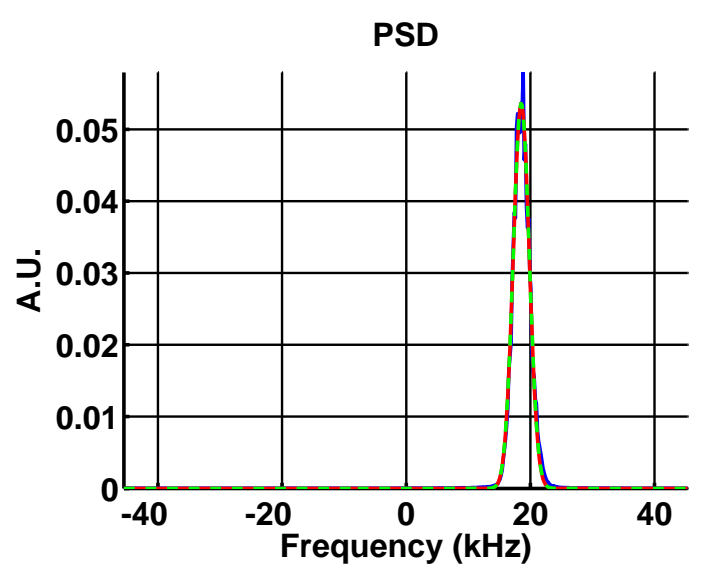

(a) PSD

$\operatorname{Re}(\mathrm{R})$

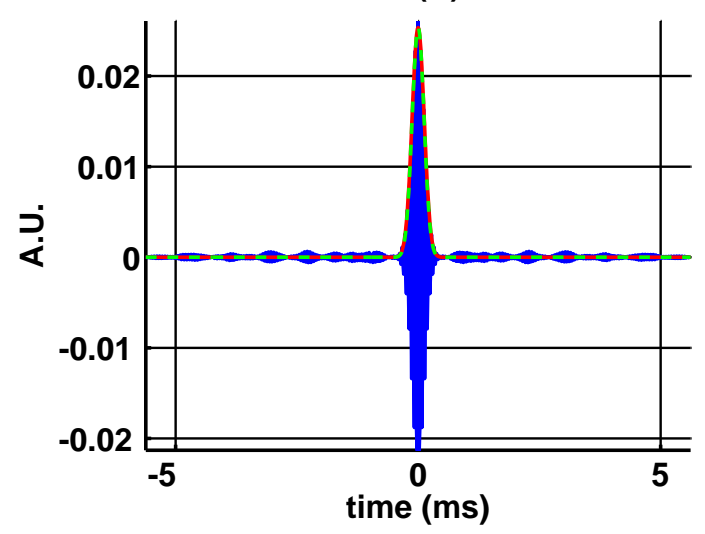

(c) $\operatorname{Re}(R)$

Experimental Fit

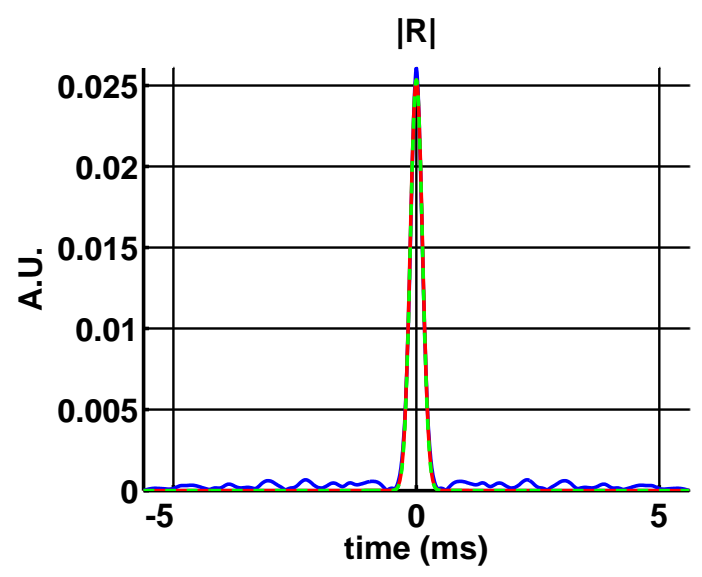

(b) $|R|$

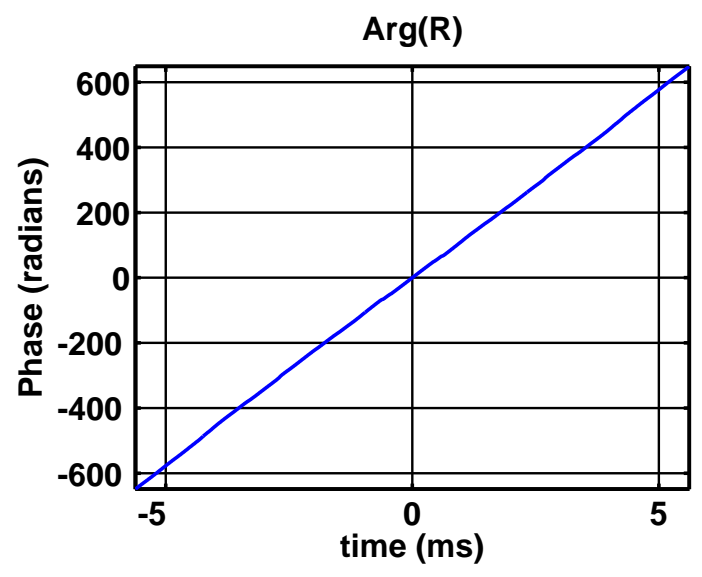

(d) $\arg (R)$

Experimental Data Theoretical

Figure 3: Temporal PSD and autocorrelation ${ }^{11,12}$ measured from a $50 \mu \overline{\mathrm{L} \cdot \mathrm{min}^{-1}}$ intralipid flow phantom. (a) The PSD shows a Doppler shift due to the axial component of intralipid flow velocity. (b) The absolute value of the autocorrelation function, like the PSD, is Gaussian. (c) The real part of the autocorrelation function is equal to the absolute value of the autocorrelation multiplied by a cosine function as determined by the Doppler shift $\cos \left(4 \pi n v_{z} \tau / \lambda_{0}\right)=\operatorname{Re}\left[\exp \left(j 4 \pi n v_{z} \tau / \lambda_{0}\right)\right]$. (d) The phase (unwrapped) of the autocorrelation function indicates Doppler shift. The gradient is equal to the Doppler shift and is proportional to $v_{z}$. The green lines show the expected PSD, Eq. (7), and autocorrelation, Eq. (6), from the measured Doppler velocity of $47.2 \mathrm{~mm} \cdot \mathrm{s}^{-1}$ from this expected beam waist. The red lines, which overlap with the green lines, show the non-linear least squares best fit Gaussian curves of the experimental data.

\section{MULTIPLICATIVE DECORRELATION MAXIMUM LIKELIHOOD ESTIMATOR}

To include decorrelation noise in the signal model, Eq. (1), consider scatterers moving transversely across an OCT voxel. At any time instant, they are randomly distributed within the voxel. Over time, the configuration of scatterers changes, and the signal "decorrelates," broadening the PSD. Similarly, decorrelation can arise when scanning the OCT beam across a static sample. One can simulate this with Doppler shifted correlated random variables. Hence, we modify the signal from Eq. (1) to include a multiplicative term $q_{n}$,

$$
s_{n}=q_{n}|r| \exp [j(n \Omega \Delta t)]+z_{n},
$$

where $q_{n}$ is a correlated complex Gaussian random variable with a known auto-covariance matrix, $\boldsymbol{\Sigma}$. Here $\boldsymbol{\Sigma}$ is real and Toeplitz symmetric, with the first row having a Gaussian profile. Its $1 / e^{2}$ half-width is determined by 


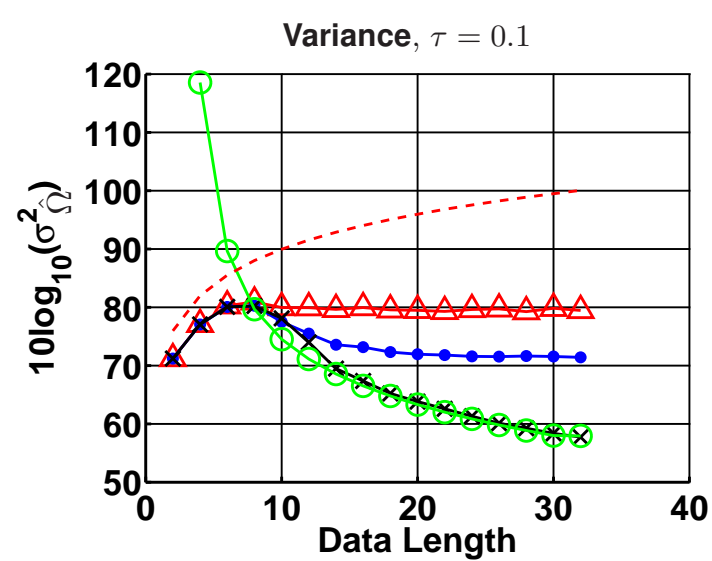

(a)

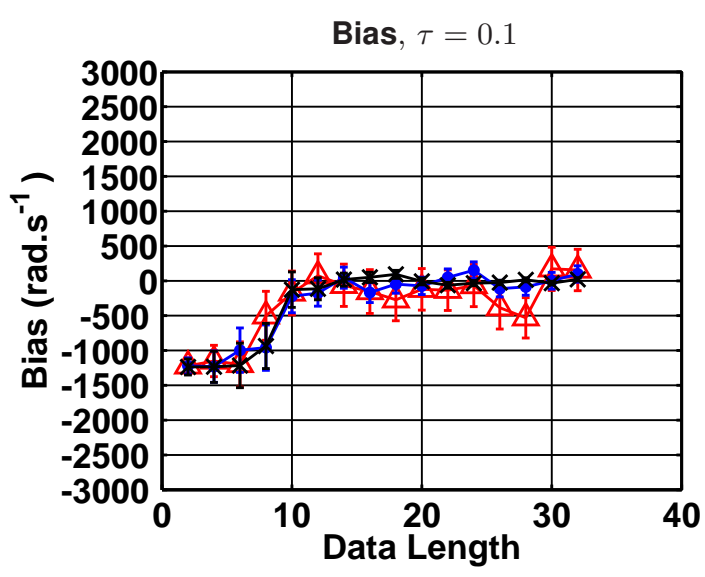

(b)

$\longrightarrow$ Decorrelation MLE- $\triangle$ AWGN MLE

- Max Var

$-\mathrm{CRLB}$

Figure 4: Increasing the acquisition rate increases the performance of the decorrelation noise MLE relative to the other estimators. ${ }^{5}$ The decorrelation MLE also achieves the Cramer-Rao lower bound (CRLB). This figure shows the estimators under simulated multiplicative decorrelation noise and negligible additive noise against data length for a constant acquisition time. Here, the acquisition time is $1 \mathrm{~ms}$, and 1000 repetitions were acquired. A Gaussian weighted covariance matrix was used, with a coherence time of $0.10 \mathrm{~ms}$. Hence $\Delta t / \tau$ ranged from 5.000 down to 0.3125 . At short data lengths, decorrelation leads to aliasing or "phase wrapping"; however the Doppler shift estimate was constrained to lie within the bounds determined by the sampling rate. The maximum variance for an estimator, taking into account phase wrapping, is given by $N^{2} \pi^{2} / T^{2}$. (a) Variances are measured in $\operatorname{rad}^{2} \cdot \mathrm{s}^{-2}$. (b) Estimator bias in radians per second.

the coherence time of the signal. In our earlier work, ${ }^{5}$ we have shown that as additive white noise is statistically unchanged under Doppler shifts, it may be included in $q_{n}$ through decreasing the off diagonal terms in the covariance matrix $\boldsymbol{\Sigma}$ by an amount determined by the signal to white noise ratio (SWNR). Hence we may set $z_{n}=0$ without loss of generality. The likelihood function is given by ${ }^{5}$

$$
\hat{\Omega}_{\mathrm{dMLE}}=\underset{\Omega}{\arg \min }\left(\mathbf{s}^{T}(\Omega) \mathbf{\Sigma}^{-1} \mathbf{s}(\Omega)\right)
$$

where $\mathbf{s}=\left(s_{1} \exp (-j \Omega \Delta t), \ldots, \quad s_{N} \exp (-j N \Omega \Delta t)\right)^{T}$. As we have shown, decorrelation is an inherent property of OCT signals with flow, and hence this estimator is expected to perform better than the AWGN MLE. We have shown that with an appropriate covariance matrix, the decorrelation noise MLE reduces to the AWGN MLE. ${ }^{5}$ Hence the decorrelation noise MLE is a more general estimator than the AWGN MLE. This MLE formalism may be used to estimate the coherence time, $\tau$, if $\boldsymbol{\Sigma}(\tau)$ is an explicit function of the coherence time.

\section{KASAI ESTIMATOR}

We use the Kasai estimator ${ }^{9}$ in its discrete form. The phase subtended, $\angle$, by the lag one autocorrelation function over the time between measurements, $\Delta t$, gives the Doppler frequency estimate,

$$
\hat{\Omega}_{\text {Kasai }}=\frac{L(\hat{R}(\Delta t))}{\Delta t}=\frac{L\left(\sum_{n=1}^{N-1} s_{n+1} s_{n}^{*}\right)}{\Delta t}=\frac{L\left\{\sum_{n=1}^{N-1}\left|s_{n+1}\right|\left|s_{n}\right| \exp \left[j\left(\phi_{n+1}-\phi_{n}\right)\right]\right\}}{\Delta t} .
$$

Here $s_{n}$ is the signal acquired at the $n$th time instant, and $\phi_{n}$, its phase. As no assumptions are made about the noise statistics, the Kasai method provides reasonable estimates even in the presence of decorrelation noise. ${ }^{4}$

\section{COMPARISON OF PERFORMANCE}

We ran simulations to estimate the variances and biases of the estimators. The analog frequency was assumed to be $\Omega=400 \pi$ rad.s $^{-1}$ for all simulations, without loss of generality. The simulated acquisition rates were well 


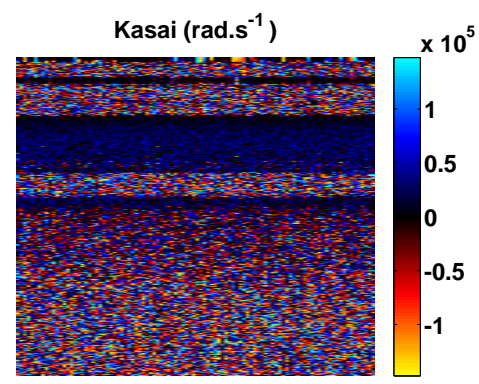

(a) Kasai estimator

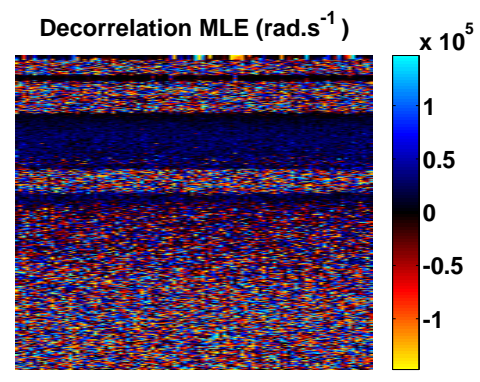

(b) Decorrelation MLE

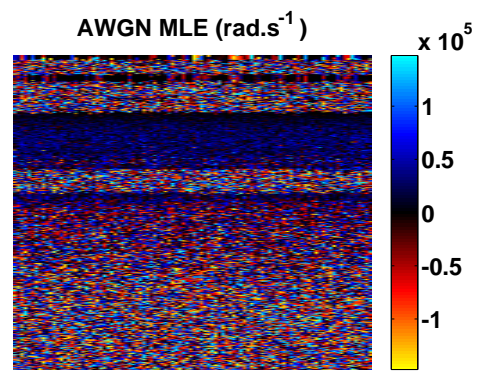

(c) AWGN MLE

Figure 5: Color Doppler Maps of $3 \mathrm{ml}_{\mathrm{hr}}{ }^{-1} \mathrm{RBC}$ flow, through $0.58 \mathrm{~mm}$ inner diameter tubing, with an average axial velocity of $0.91 \mathrm{~mm} \cdot \mathrm{s}^{-1}$ (Doppler frequency measurements in rad. $\mathrm{s}^{-1}$ ). The line scan rate is $47 \mathrm{kHz}$, with a data length of 8. Under these conditions, the Kasai estimator has an estimation variance of $2.70 \times 10^{8} \mathrm{rad}^{2} . \mathrm{s}^{-2}$, or $84.3 \mathrm{~dB}$. The decorrelation MLE shows a $0.72 \mathrm{~dB}$ improvement over the Kasai estimator, with a variance of $2.29 \times 10^{8} \mathrm{rad}^{2} . \mathrm{s}^{-2}$. The AWGN MLE performance is $3.43 \mathrm{~dB}$ worse than the Kasai estimator's, with a variance of $5.95 \times 10^{8} \mathrm{rad}^{2} . \mathrm{s}^{-2}$. The $1 / e^{2}$ half width coherence time is approximately $4.6 \Delta t \approx 0.1 \mathrm{~ms}$.

above the Nyquist limit as the lowest simulated acquisition rate was $2 \mathrm{kHz}$. Multiplicative decorrelation noise in the signal simulates the presence of moving scatterers. Fig. 4 shows that under the presence of multiplicative noise, and negligible additive noise, the decorrelation noise MLE has the best performance, and the AWGN MLE has the worst performance. As the acquisition rate and data length increase, the relative improvement of the decorrelation MLE also increases. Similarly, increasing the coherence time increases the relative performance improvement of the decorrelation MLE. ${ }^{5}$

In Figs. 5 and 6 a $1310 \mathrm{~nm}$ spectral/Fourier domain OCT microscope was used for imaging. The light source consisted of two super-luminescent diodes combined by using a 50/50 fiber coupler to yield a spectral bandwidth of $170 \mathrm{~nm}$. The axial (depth) resolution was $3.6 \mu \mathrm{m}$, full-width-at-half-maximum (FWHM), and the transverse resolution was $7.2 \mu \mathrm{m}$ FWHM, and the highest imaging speed was 47000 axial scans per second, achieved by an InGaAs line scan camera (Goodrich-Sensors Unlimited, Inc). The camera sensitivity was typically set to "medium" to obtain the widest dynamic range. A $5 \times$ objective, Mitsutoyu, was used and the center of tubing was placed in focus. We used a syringe pump with $0.58 \mathrm{~mm}$ inner diameter tubing and rat blood, using heparin as the anticoagulant. The tube was placed at a $16^{\circ}$ incline, so that there was an axial velocity which could be measured as a Doppler shift. Due to the Poiseuille flow profile of the tube, measurements of the Doppler shift were taken at $0.15 \mathrm{~mm}$ from the inner edge of the tubing for consistency when making comparisons. The estimation variances were computed from 100 estimates. To compute the decorrelation MLE, the auto-covariance function was estimated from data. The PSD half-width $1 / e^{2}$ maxima were estimated from data using least squares fitting. The DFT length of the AWGN MLE was increased by 256 times using zero padding, so that the estimator variance would not be artifactually rounded to zero.

Fig. 5 shows that for a $3.0 \mathrm{ml} . \mathrm{hr}^{-1}$ flow rate, the decorrelation noise MLE has the best performance, whereas the AWGN MLE performed more poorly. Images of the rat retina, Fig. 6, also suggest that the decorrelation noise MLE performs better than the AWGN MLE and the Kasai estimator. We have shown in earlier work that the decorrelation MLE can outperform the AWGN MLE in all conditions, as it is a more general ML estimator, and the covariance matrix can be designed to incorporate additive noise into the estimation model. ${ }^{5}$

\section{CONCLUSION}

We have shown that while the AWGN MLE is statistically optimal under additive white Gaussian noise conditions observed in slow flow, decorrelation is an inevitable consequence of signals acquired from moving scatterers, such as when there is relative movement across an OCT beam. Therefore a AWGN model is inadequate to describe the signal. As a consequence, the AWGN MLE becomes statistically suboptimal when used to quantify faster flow. We have derived a decorrelation noise MLE that is more general than the AWGN MLE and can incorporate 


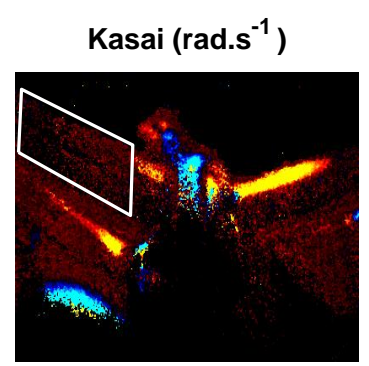

(a) Kasai estimator

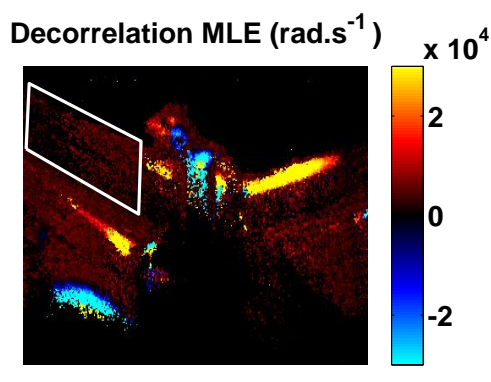

(b) Decorrelation MLE

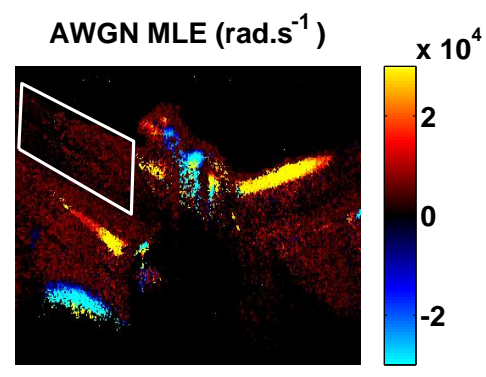

(c) AWGN MLE

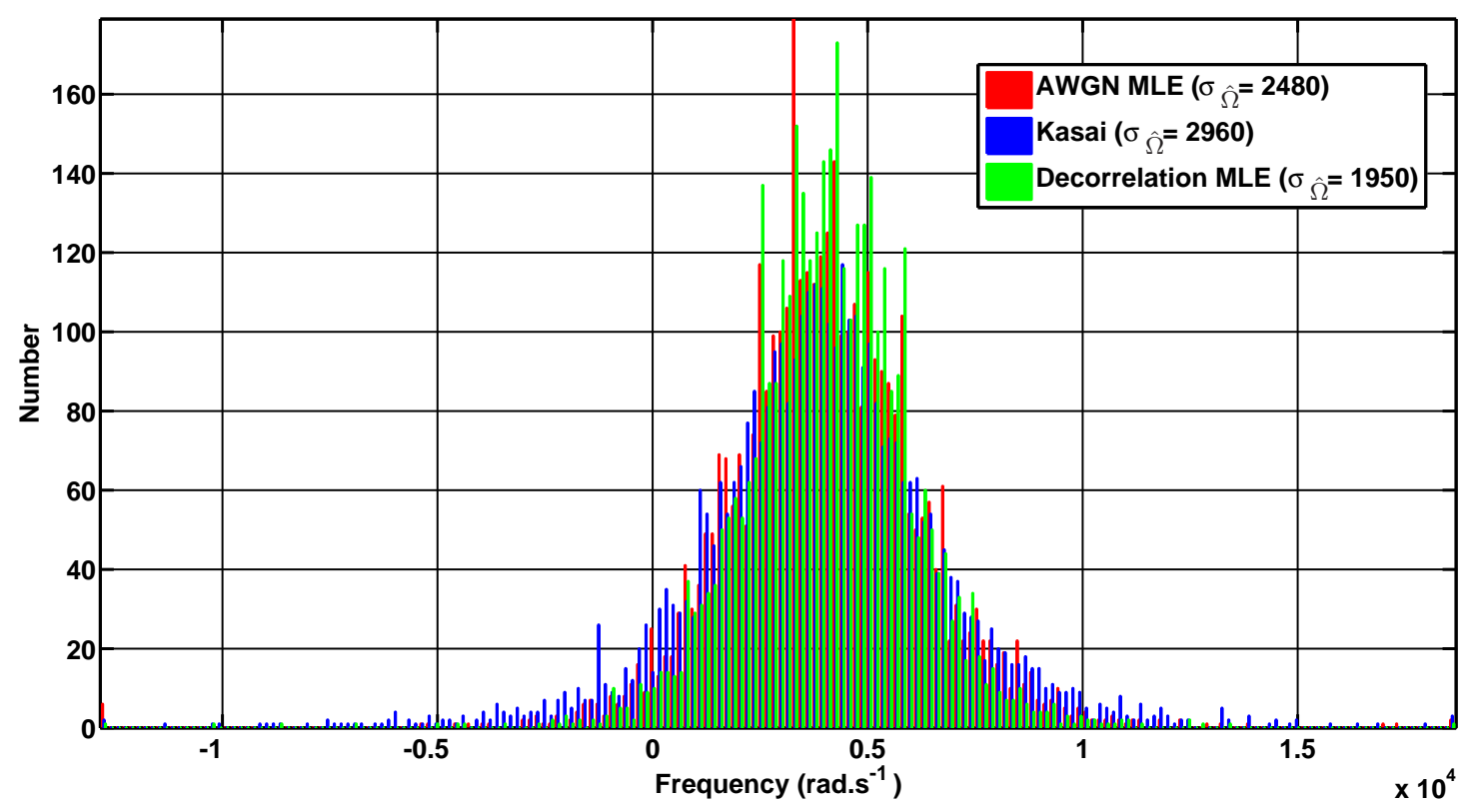

(d) Histogram of Doppler Maps

Figure 6: Doppler maps of a rat retina using (a) Kasai, (b) Decorrelation MLE, and (c) AWGN MLE algorithms. Data was acquired at an A-line rate of $47 \mathrm{kHz}$, and estimates were obtained from blocks of 20 transverse pixels. (d) Histogram of Doppler shifts in the regions shown in (a)-(c). The decorrelation MLE has a narrower distribution of estimates, suggesting that it is a more precise estimator.

decorrelation noise as well as AWGN into the estimation model. We have shown that the decorrelation noise MLE has better performance than the AWGN MLE and the Kasai estimator. We have also shown that while decorrelation reduces the performance of the AWGN MLE, it has a well defined linear relationship with Doppler shift for point scatterers. Decorrelation itself, taking into account the more complicated scattering effects for red blood cells, may be used as a measure of flow speed in vasculature, in a well-calibrated system where the axial and transverse resolutions are known. Decorrelation methods complement Doppler-based approaches and potentially quantify nearly transverse flow where Doppler shifts are too small to measure.

\section{ACKNOWLEDGMENT}

We acknowledge support from the Fulbright Scholarship Board, the Lee-Hysan Foundation, the Hong Kong Research Grants Council projects 7138/11E and 7131/12E, the University Research Committee of The University of Hong Kong project 104002345, the National Institutes of Health (R00NS067050, EB001954 R01), the American Heart Association (11IRG5440002), and the Glaucoma Research Foundation Catalyst for a Cure. 


\section{REFERENCES}

[1] Huber, R., Adler, D. C., and Fujimoto, J. G., "Buffered fourier domain mode locking: unidirectional swept laser sources for optical coherence tomography imaging at 370,000 lines/s," Opt. Lett. 31, 2975-2977 (October 2006).

[2] Srinivasan, V. J., Adler, D. C., Chen, Y., Gorczynska, I., Huber, R., Duker, J. S., and Fujimoto, J. G., "Ultrahigh-speed optical coherence tomography for three-dimensional and en face imaging of the retina and optic nerve head," Invest. Ophthalmol. Vis. Sci. 49, 5108-5110 (November 2008).

[3] Chan, A. C., Lam, E. Y., and Srinivasan, V. J., "Comparison of kasai autocorrelation and maximum likelihood estimators for doppler optical coherence tomography," IEEE Trans. Med. Imag. 32, 1033-1042 (June 2013).

[4] Vakoc, B., Tearney, G., and Bouma, B., "Statistical properties of phase-decorrelation in phase-resolved doppler optical coherence tomography," IEEE Trans. Med. Imag. 28, 814-821 (June 2009).

[5] Chan, A. C., Lam, E. Y., and Srinivasan, V. J., "Maximum likelihood doppler frequency estimation under decorrelation noise for quantifying blood flow in optical coherence tomography," IEEE Trans. Med. Imag. (accepted pending revisions) (2014).

[6] Szkulmowski, M., Szkulmowska, A., Bajraszewski, T., Kowalczyk, A., and Wojtkowski, M., "Flow velocity estimation using joint spectral and time domain optical coherence tomography," Opt. Express 16, 6008-6025 (April 2008).

[7] Szkulmowska, A., Szkulmowski, M., Kowalczyk, A., and Wojtkowski, M., "Phase-resolved doppler optical coherence tomography - limitations and improvements," Opt. Lett. 33, 1425-1427 (July 2008).

[8] Schmoll, T., Kolbitsch, C., and Leitgeb, R. A., "Ultra-high-speed volumetric tomography of human retinal blood flow," Opt. Express 17, 4166-4176 (March 2009).

[9] Kasai, C., Namekawa, K., Koyano, A., and Omoto, R., "Real-time two-dimensional blood flow imaging using an autocorrelation technique," IEEE Trans. Sonics Ultrason. 32, 458-464 (May 1985).

[10] Makita, S., Hong, Y., Yamanari, M., Yatagai, T., and Yasuno, Y., "Optical coherence angiography," Opt. Express 14, 7821-7840 (Aug 2006).

[11] Srinivasan, V. J., Radhakrishnan, H., Lo, E. H., Mandeville, E. T., Jiang, J. Y., Barry, S., and Cable, A. E., "Oct methods for capillary velocimetry," Biomed. Opt. Express 3, 612-629 (March 2012).

[12] Srinivasan, V. J., Sakadžić, S., Gorczynska, I., Ruvinskaya, S., Wu, W., Fujimoto, J. G., and Boas, D. A., "Quantitative cerebral blood flow with optical coherence tomography," Opt. Express 18, 2477-2494 (February 2010).

[13] Imai, Y. and Tanaka, K., "Direct velocity sensing of flow distribution based on low-coherence interferometry," J. Opt. Soc. Am. A 16, 2007-2012 (August 1999). 